\title{
Numerical Experiments on the Genesis and Development of Typhoon Influenced by the Momentum Flux due to Environmental Flow
}

\author{
By Qiushi Chen \\ Department of Geophysics, Peking University, Beijing, People's Republic of China \\ (Manuscript received 30 October 1986, in revised form 23 February 1987)
}

\begin{abstract}
A nine-level primitive equation axisymmetric model is used to simulate the genesis and development of typhoon influenced by environmental flow. In an experiment $A$, the wind field and the horizontal gradients of the temperature, geopotential height, surface presure and moisture field all vanish in the initial state, but there is a specified source of cyclonic momentum in the upper troposphere. This acceleration is physically related to an observed eddy flux forcing term due to asymmetries in the environmental flow. A typhoon develops very rapidly from this initial state with some other usual conditions.

The dynamical essentials of the influence of the environmental flow is further illustrated by an experiment B. If an unbalanced vortex, which is hypothesized to occur as a result of the eddy flux of momentum, is used as an initial values instead of the forcing term in the governing equations, a typhoon develops more quickly. Some relationship between the results from the present paper and CISK theory is discussed.
\end{abstract}

\section{Introduction}

In a synoptic analysis study of 15 typhoon cases by Chen and Zhong (1965), it was found that the absolute vorticity advection of the large-scale environmental flow at the $200 \mathrm{mb}$ level was larger than in the lower levels in the region before the formation of typhoons. Black and Anthes (1971), in an analysis of five tropical cyclones, also indicated that much of the outward eddy transport of the anticyclonic vorticity was taking place in the upper outflow jets, during the development of the hurricanes. This eddy transport recently was further confirmed by Holland (1983a) from an angular momentum budget study for tropical cyclones. The distribution of the increases in cyclonic tangential wind resulted from the eddy flux of angular momentum in the composite intensifying tropical cyclone (WPD3) by Holland (1983a) is shown in Fig. 1.1. Dashed lines denotes the inflow layer between surface and $850 \mathrm{mb}$ level and the solid line denotes the outflow layer between 250 and $100 \mathrm{mb}$. From Fig. 1.1, we can see that the increase in cyclonic tangential wind in the upper outflow layer is larger than that in the lower inflow layer in WPD3.

Recently we analysed some cases of the development of typhoons in the tropical west Pacific based on the FGGE IIIb data set. It was also found that the positive vorticity advection in upper levels was larger than that in lower levels $24 \mathrm{~h}$ before typhoon formotion. As an example, a low-level disturbance developed into a typhoon on 00GMT 17, August 1979. The surface pressure was $978 \mathrm{mb}$ and maximum wind velocity was about 20 $\mathrm{m} / \mathrm{sec}$ at that time. Fig. $1.2(\mathrm{a})$, (b) and (c) are the streamlines, horizontal_vorticity advection, $-V \cdot \nabla(f+\zeta)$, on $300 \mathrm{mb}$ level and the difference of vorticity advection between 300 $\mathrm{mb}$ and $850 \mathrm{mb}$ level on 00GMT 16, Aug. 


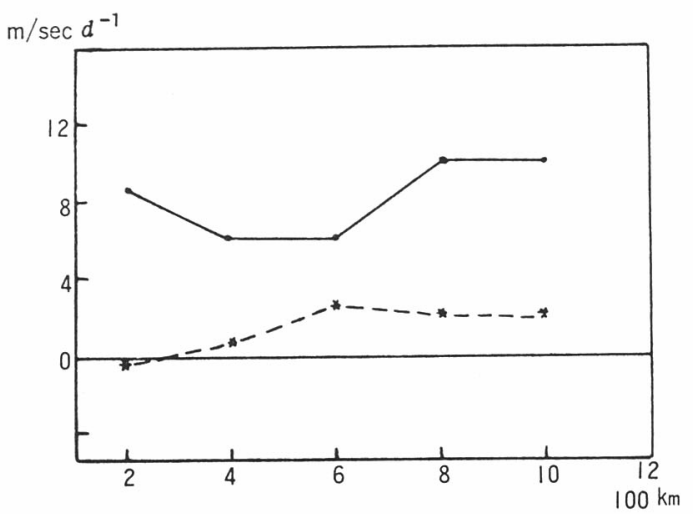

Fig. 1.1 The distribution of the increase in cyclonic tangential speed resulting from the eddy flux of angular momentum in intensifying cyclones (WPD3) by Holland (1983a). Dashed line denotes inflow (surface-850mb) layer and solid line denotes outflow (250-100 $\mathrm{mb}$ ) layer.

1979, respectively. From Fig. 1.2 (c) it can be seen that there is a large positive value of the difference of the vorticity advection between the 300 and $850 \mathrm{mb}$ level over the region $24 \mathrm{~h}$ before the formation of the typhoon. The variations with time of the vorticity advection on 300 and $850 \mathrm{mb}$ level are shown in Table 1 over the center of the surface disturbance of next $24 \mathrm{~h}$.

It can be seen from Table 1 that the differences of vorticity advection between 300 and $850 \mathrm{mb}$ level are larger than $2 \times 10^{-10} \mathrm{sec}^{-2}$ before the formation of typhoon, but after the typhoon formation the difference becomes negative.

The above results can be understood by an analysis using the splitting method. Chen (1964) proposed a splitting method to study the mechanism for the formation of the typhoon, especially in the early stage. The variation of the large scale motion can be divided into a series of short time intervals. The variations and their governning equations in each short time interval are split into two parts: The advection process is described approximately by

$$
\begin{aligned}
& \left(\frac{\partial \zeta}{\partial t}\right)_{a d v}=-V \cdot \nabla(f+\zeta) \\
& \frac{\partial}{\partial t}\left(\frac{\partial \phi}{\partial p}\right)_{a d v}=-V \cdot \Gamma\left(\frac{\partial \phi}{\partial p}\right)
\end{aligned}
$$
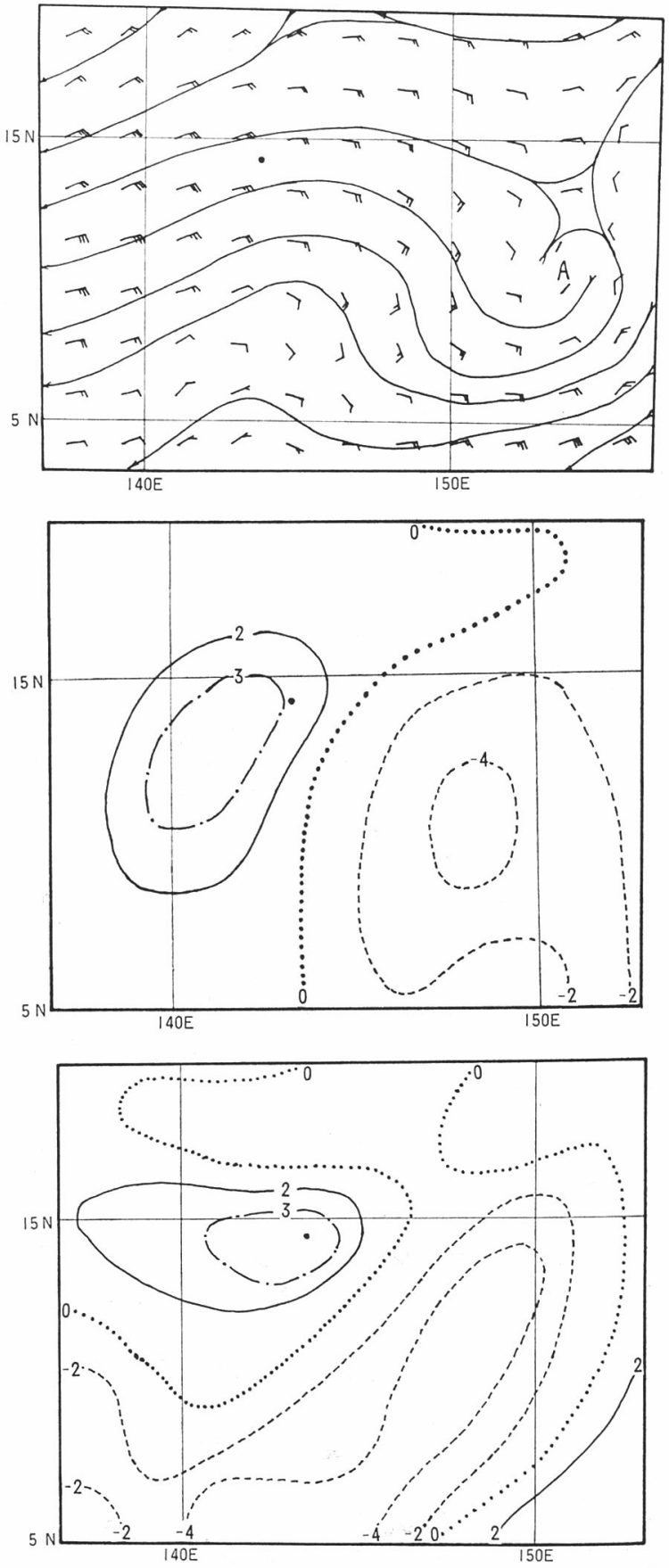

Fig. 1.2 (a) streamlines on $300 \mathrm{mb}$ level, (b) contours of the horizontal advection of absolute vorticity, $-V \cdot \nabla(f+\zeta)$, on $300 \mathrm{mb}$ level (Unit $10^{-10} \mathrm{sec}^{-2}$ ) and (c) contours of the difference of the absolute vorticity advection between 300 and $850 \mathrm{mb}$ levels, $-\left.V \cdot \nabla(f+\zeta)\right|_{850 \mathrm{mb}} ^{300 \mathrm{mb}}$, on O0GMT, 16 August 1979. Heavy dot denotes surface position of typhoon next $24 \mathrm{~h}$. 
Table 1. The vorticity advections on 300 and $850 \mathrm{mb}$ level and their differences over the center of the surface disturbance of next $24 \mathrm{~h}$, (unit: $10^{-10} \mathrm{sec}^{-2}$ ).

\begin{tabular}{lcccrrr}
\hline & Aug. 15 & 15 & 16 & 16 & 17 & 17 \\
& 00GMT & $12 \mathrm{GMT}$ & 00GMT & 12GMT & 00GMT & 12GMT \\
\hline$-\left.V \cdot P(f+\zeta)\right|_{300 \mathrm{mb}}$ & 3.7 & 1.6 & 2.9 & 2.4 & 0 & 0 \\
$-\left.V \cdot P(f+\zeta)\right|_{850 \mathrm{mb}}$ & 0.8 & -0.6 & -1.0 & -0.2 & 15.5 & 3.8 \\
$-\left.V \cdot \nabla(f+\zeta)\right|_{850 \mathrm{mb}} ^{300 \mathrm{~b}}$ & 2.6 & 2.2 & 3.9 & 2.6 & -15.5 & -3.8 \\
\hline
\end{tabular}

and the adjustment process is described approximately by

$$
\begin{aligned}
& \left(\frac{\partial \zeta}{\partial t}\right)_{a d j}=-f \nabla \cdot V \\
& \left(\frac{\partial \nabla \cdot V}{\partial t}\right)_{a d j}=f \zeta-\nabla^{2} \phi \\
& \frac{\partial}{\partial t}\left(\frac{\partial \phi}{\partial p}\right)_{a d j}=-\frac{C^{2}}{p^{2}} \omega-\frac{R}{p C_{p}} Q_{\text {cond }}
\end{aligned}
$$

where $Q_{\text {cond }}$ is the heating rate of condensation for unit mass,

$$
C^{2}=\frac{R^{2} T}{g}\left(r_{d}-r\right), \quad \omega=\frac{d p}{d t},
$$

other symbols are used as usual.

It

$$
\frac{\partial}{\partial t}\left(-\frac{\partial \zeta}{\partial p}\right)_{a d v}-\frac{\partial}{\partial t} \frac{1}{f}\left(-\nabla^{2} \frac{\partial \phi}{\partial p}\right)_{a d v} \neq 0
$$

in a short time interval, a thermal wind imbalance will develop by the advection process, even if the thermal wind balance is satisfied at the initial moment of this interval. This imbalance can be called an implicit imbalance, and it can only be deduced analytically in the advection process based on the splitting method. The imbalance produced by the advection process will cause a vertical circulation to adjust towards a new balance. If the horizontal advection of absolute vorticity is greater at $300 \mathrm{mb}$ than at $850 \mathrm{mb}$ and the temperature advection is very small, an imbalance in which

$$
\zeta_{T}^{\prime}=\left(-\frac{\partial \zeta}{\partial p}\right)-\nabla^{2}\left(-\frac{\partial \phi}{\partial p}\right)>0,
$$

the vorticity of the vertical shear wind is larger than the thermal vorticity, will be produced by the advection process. Chen
(1964) found that, in a conditionally unstable and sufficiently moist atmosphere, if the horizontal scale of the perturbation is smaller than a characteristic scale Lo, the inertiagravity wave is unstable in the adjustment process. A positive feedback between this imbalance and a vertical circulation with the convergence in the lower and the divergence in the upper troposphere will occur. A vortex with cyclonic circulation in the lower level and anticyclonic in the upper grows very rapidly. This instability is not a free convection induced by thermal buoyant force, but is forced by the adjustment process due to the imbalance introduced by the advection process.

Many numerical models have simulated the life cycle of the hurricanes with reasonably success based on the CISK mechanism. The initial values used in the modeling based on CISK are usually a balanced surface vortex. The intensity of the initial cyclones assumed by modelers, according to Gray (1977), is

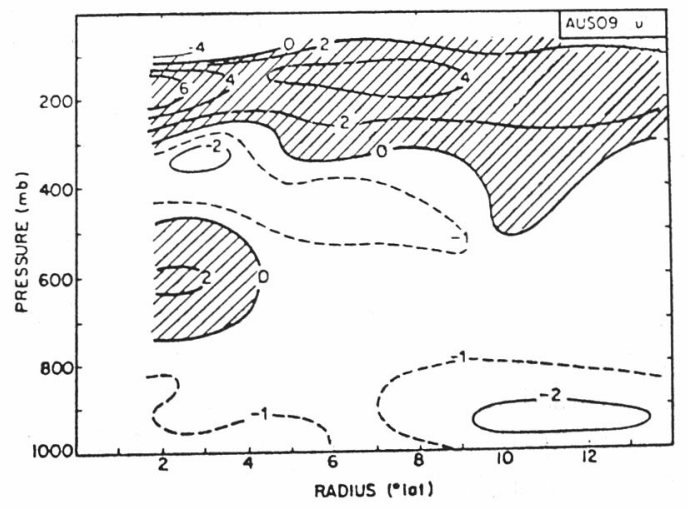

Fig. 1.3 Axisymmetric vertical cross-section of radial winds $(\mathrm{m} / \mathrm{s})$ for the composite intensifying tropical strom (AUS09) (after Holland 1983b). 
usually stronger than observed typical precyclone disturbances. From the vertical distribution of mass inflow in the observed intensifying composite typhoon by Holland (1983b) shown in Fig. 1.3, it can be seen that except a maxima of mass inflow in the boundary layer, there is a secondary maxima near $400 \mathrm{mb}$ level. This secondary maxima of mass inflow is very difficult to explain by the boundary layer friction in the CISK mechanism.

In order to illustrate how the large-scale environmental cirulation affects the genesis and development of the typhoon, a series of numerical experiments have been carried out by an axisymmetric model. The model used to simulate simultaneously the circulation of a typhoon itself and its environment should be an asymmetric, three-dimensional model. However, we use an axisymmetric model as a first step. Because the scale of the environmental flow is larger than the typhoon, the horizontal angular momentum advection of the environmental flow can only be described by an eddy flux of the momentum in the axisymmetric model. Thus a forcing term denoting the convergence of the eddy momentum flux will appear in the tangantial momentum equation. Challa and Pfeffer (1980) studied the effects of the eddy flux of angular momentum on model hurricane development by means of Sundqvist (1970)'s quasi-balanced model. Sundqvist (1970), using a quasibalanced model, simulated the process of the hurricane development, from an initial balanced vortex with a maximum tangential speed about $15 \mathrm{~m} / \mathrm{s}$. Challa and Pfeffer (1980) found that, if the angular momentum flux convergences with magnitudes comparable to those observed value are introduced into the model, Sundqvist (1970)'s model hurricane development can be markedly accelerated. Hewever, in present paper, no any balanced vortex is used in the initial condition, which is essentially different from that in Challa and Pfeffer (1980)'s paper.

In the present paper, the typhoon development will be simulated from an idealized initial state in which the wind field and the horizontal gradients of the temperature, geopotential height, surface pressure and moisture fields all vanish. Thus the typhoon development in the model can only be triggered by the influence of environmental flow.

Yamasaki (1977) and Rosenthal (1978) avoided using the parameterization method of cumulus convection in their modls. Rosenthal (1978) computed the latent heat release on the resolvable scale on a $20 \mathrm{~km}$ horizontal grid to simulate the development of a hurricane successfully from an initial balanced vortex with surface wind about $12 \mathrm{~m} / \mathrm{s}$. In the present paper, only explicit, resolvable-scale condensation of water vapor is considered. The model does not carry liquid water as a dependent variable, so that it is simpler than Rosenthal (1978)'s model.

\section{Basic equations}

The governing equations are formulated by an $f$-plane system using axisymmetric cylindrical coordinates in the horizontal and $\sigma$ coordinate in the vertical. We define $\sigma$ as

$$
\sigma=\frac{p-P_{T}}{P_{S}-P_{T}}=\frac{p-P_{T}}{\pi}
$$

where the top boundary pressure $P_{T}$ is $50 \mathrm{mb}$, and the surface pressure $P_{S}$ is a function of the horizontal coordinate and time, $P_{S}(R, \sigma, t)$. Let $R_{0}$, the outer boundary point of the typhoon is the original along the radial direction, i.c. the radial coordinate is, $R=R_{0}-r$. At the center of the typhoon, $r=0$ and $R=R_{0}$. and at the lateral bounday, $r=R_{0}$ and $R=0$. The integreted interval in the radial direction is that $0 \leqq R \leqq R_{0}$.

The horizontal momentum equation can be written as

$$
\begin{aligned}
& \frac{\partial u}{\partial t}+v \frac{\partial r u}{r \partial R}+\dot{\sigma} \frac{\partial u}{\partial \sigma}-f v=-\frac{\partial \dot{\pi} r^{2} \overline{u^{\prime} v^{\prime}}}{\pi r^{2} \partial R}-S u \\
& \frac{\partial v}{\partial t}+v \frac{\partial v}{\partial R}+\dot{\sigma} \frac{\partial v}{\partial \sigma}+\left(f+\frac{u}{r}\right) u-\left(\frac{\partial \phi}{\partial R}\right)_{p}=S v
\end{aligned}
$$

where $u$ is the tangential component of the wind (positive in the direction of anti-clockwise) and $v$ is the radial component (positive inward $).(\partial / \partial R)_{p}$ represents derivatives along 
the isobaric surface. The overbar, "- " denotes an azimuthal mean and prime, a deviation from this mean. $f$ is a constant and taken to be a value for $18^{\circ}$ latitude. $S u$ and $S v$ are the friction due to small-scale turbulence. The mass continuity equation, the thermodynamic equation and water vapor mass continuity equation can be written

$$
\begin{aligned}
& \frac{\partial \pi}{\partial t}+\frac{1}{r} \frac{\partial(\pi v r)}{\partial R}+\frac{\partial(\pi \dot{\sigma})}{\partial \sigma}=0 \\
& \frac{\partial \theta}{\partial t}+v \frac{\partial \theta}{\partial R}+\dot{\sigma} \frac{\partial \theta}{\partial \sigma}=\frac{1}{C p P}[L(C-E)+Q] \\
& \frac{\partial q}{\partial t}+v \frac{\partial q}{\partial R}+\dot{\sigma} \frac{\partial q}{\partial \sigma}=-(C-E)+S q
\end{aligned}
$$

where $\theta=T / P$ is potential temperature, $P=$ $\left(p / p_{0}\right)^{\kappa}, p_{0}=1000 \mathrm{mb}, \kappa=R / C p, R$ is gas constant of air and $C p$ is specific heat capacity of air. $C$ and $E$ are the rate of resolvablescale condensation and evaporation per unit mass of dry air, $Q$ is the heating rate per unit mass by the turbulent transfer and the radiation. The hydrostatic equation is

$$
d \Phi=-C p \theta d P
$$

The upper and lower boundary condition are given by

$$
(\pi \dot{\sigma})_{\sigma=0}=0 \text { and }(\pi \dot{\sigma})_{\sigma=1}=0
$$

The nonlinear horizontal and vertical eddy diffusion processes can be written as

$$
\begin{aligned}
& S u=\frac{1}{\pi r}\left[\frac{\partial}{\partial R}\left(\pi K_{H} r \frac{\partial u}{\partial R}\right)-\frac{\pi K_{H} u}{r}\right]+\frac{g}{\pi} \frac{\partial \tau_{u}}{\partial \sigma} \\
& S v=\frac{1}{\pi r}\left[\frac{\partial}{\partial R}\left(\pi K_{H} r \frac{\partial v}{\partial R}\right)-\frac{\pi K_{H} v}{r}\right]+\frac{g}{\pi} \frac{\partial \tau_{v}}{\partial \sigma} \\
& \frac{Q}{C p P}=\frac{1}{\pi r}\left[\frac{\partial}{\partial R}\left(\pi K_{H} r \frac{\partial \theta}{\partial R}\right)\right] \\
& +\frac{1}{C p P}\left[\frac{g}{\pi} \frac{\partial F_{h}}{\partial \sigma}+Q_{R}\right] \\
& S q=\frac{1}{\pi r}\left[\frac{\partial}{\partial R}\left(\pi K_{H} r \frac{\partial q}{\partial R}\right)\right]+\frac{g}{\pi} \frac{\partial F_{q}}{\partial \sigma}
\end{aligned}
$$

The lateral eddy mixing coefficient is written as

$$
K_{H}=K_{H_{0}}+l_{\bar{H}}^{\circ}|D|
$$

The quantity $|D|$ is the magnitude of the total deformation field defined as

$$
|D|=r\left[\left(\frac{\partial}{\partial R}\left(\frac{u}{r}\right)\right)^{2}+\left(\frac{\partial}{\partial R}\left(\frac{v}{r}\right)\right)^{2}\right]
$$

The characteristic horizontal length scale $l_{H}$ is given by

$$
l_{H}=k_{0} \Delta R
$$

A value of 0.2 is used for the parameter $k_{0}$ and $K_{H} \sim 5.0 \times 10^{3} \mathrm{~m}^{2} / \mathrm{s}$.

The turbulent fluxes of momentum are given by

$$
\begin{gathered}
\tau_{u}=\frac{K_{v} g \rho}{\pi} \frac{\partial u}{\partial \sigma} \\
\tau_{v}=\frac{K_{v} g \rho}{\pi} \frac{\partial v}{\partial \sigma}
\end{gathered}
$$

where $\rho$ is the density and $K_{v}$ is the vertical eddy mixing coefficient defined as

$$
K_{v}=K_{v_{0}}+l_{v}^{2} \frac{g \rho}{\pi}\left|\frac{\partial V}{\partial \sigma}\right|
$$

A constant vertical mixing length of $30 \mathrm{~m}$ is chosen for $l_{v}$ and $K_{v_{0}} \sim 1 \mathrm{~m}^{2} / \mathrm{s}$.

All surface energy exchanges parameterized by the bulk aerodynamic method. The surface stress is given by

$$
\begin{aligned}
& \left(\tau_{u}\right)_{s}=-\rho_{s} C_{D}\left|V_{s}\right| u_{s} \\
& \left(\tau_{v}\right)_{s}=-\rho_{s} C_{D}\left|V_{s}\right| v_{s}
\end{aligned}
$$

The flux of dry static energy from the sea surface is given by

$$
\left(F_{h}\right)_{s}=C_{p} \rho_{s} C_{H}\left|V_{s}\right|\left(T_{s e a}-T_{s}\right)
$$

and the flux of total water vapor by

$$
\left(F_{q}\right)_{s}=\rho_{s} C_{H}\left|V_{s}\right|\left(q^{*}\left(T_{\text {sea }}, p_{s}\right)-q_{s}\right)
$$

where $T_{\text {sea }}$ is sea surface temperature and $q^{*}$, the saturated mixing ratio. $T_{s}$ and $q_{s}$ are the temperature and mixing ratio of the surface air. The exchange coefficients for momentum, $C_{D}$, and for heat and moisture, $C_{H}$, are 0.0022 and 0.003 respectively. Radiative heating, $Q_{R}$, is neglected.

The horizontal and vertical finite differencing of above governing equations are same 


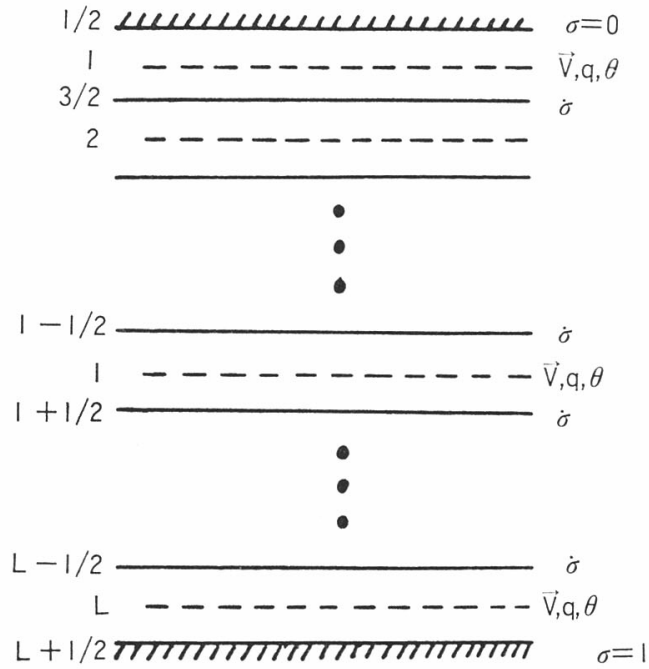

Fig. 2.1 The vertical distribution of the discrete model levels.

as that given by Chen (1987). The discrete model consists of 9 levels in the vertical direction as shown in Fig. 2.1 and 32 grid points in the horizontal direction with the grid length of $25 \mathrm{~km}$. Deep cumulus convection develops automatically in the model. In order to avoid the potential temperature decreasing with height, a dry convective adjustment is included in the model. The time differencing for governing equation is accomplished by Brown and Campara (1978)'s economic scheme coupled with time filter. A backward Matsuno (1966)'s time difference is used in the first time step. The time step $\Delta t$ used in the calculation is 30 seconds. A lateral boundary condition developed by Orlanski (1976) is used to avoid the reflection of gravity-inertia waves by lateral boundary of the model. For a more complete discussion of the model, the reader is referred to Chen (1987).

\section{Specification of initial conditions}

It has been pointed out above that the influence of the environmental flow can only be described by the eddy flux of momentum in an axisymmetric model. The convergence of the eddy momentum flux in (2.2) is written as

$$
F e=\frac{\partial \pi r^{2} \overline{u^{\prime} v^{\prime}}}{\pi r^{2} \partial R}
$$

Recently the observed distribution of the eddy momentum flux in composite typhoons under different conditions were given by Holland (1983a). The composite result WPD3 for the observed intensifying tropical cyclones was shown in Fig. 1.1. The cyclonic momentum inward in the upper outflow layer and in the outer region (more than $800 \mathrm{~km}$ from the center) are larger than that in the lower and inner region. Utilizing the condition that the eddy momentum flux vanishes in the center of the typhoon, the forcing term $F e$ can be determined by an interpolation from Fig. 1.1. The interpolated result of $\mathrm{Fe}$ is shown in Fig. 3.1. A numerical experiment in which the term $F e$ in Eq. (2.2) is used as a forcing term shown in Fig. 3.1 is denoted by Experiment A.

The initial vertical distribution of the temperature and moisture correspond to the observed mean profile within the cloud cluster in the west Pacific $\left(6^{\circ}-12^{\circ} \mathrm{N}\right)$ by Gray et al. (1975).

The initial surface pressure $P S$ is a value of $1008.7 \mathrm{mb}$. The wind field and the horizontal gradients of the temperature, geopo-

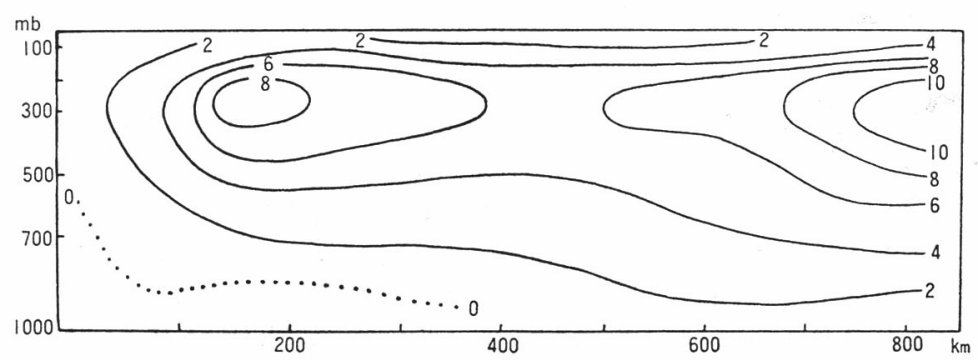

Fig. 3.1 The distribution of the acceleration of the cyclonic tangential speed by the eddy flux of angular momentum interpolated from Fig. 1.1 used in experiment A (unit: $\mathrm{m} /$ (s.d)). 
tential height, surface pressure and moisture field all vanish in the initial state. The sea surface temperature is a constant, $29^{\circ} \mathrm{C}$, in the experiment.

Based on the splitting method discussed by Chen $(1964,1981)$, the variation of the atmospheric motion can be understood by a series of interactions between the advection and adjustment processes. Using this splitting analysis method, the forcing term, $F e$, appears in the advection process and it accelerates tangental wind. Thus, an implicit imbalanced vortex, with a vertical wind shear such that the cyclonic tangential wind in upper level is larger than that in the lower will be continuously forced by $F e$ in the advection process. As we shall see later in next paragraph in the results of the experiment $\mathrm{A}$, the continuous interaction between above advection and corresponding adjustment process will cause a typhoon to generate.

In order to study the effects of the implicit imbalanced tangential wind, produced by the forcing term $\mathrm{Fe}$, on the formation of the typhoon, another experiment is designed and denoted by Experiment B. According to the theory of linear differential equations, the solution of an equation with a forcing term and with a homogeneous initial condition is equivalent to the solution of the equation with the forcing term set to be zero but with an initial condition which is obtained by a suitable time integration of the forcing term. Approximately we design experiment $\mathrm{B}$, in which the forcing term vanishes and the initial value of the tangential wind is replaced by time integretion of $\mathrm{Fe}$ for about $36 \mathrm{~h}$. As shown in Fig. 3.2, the initial tangential wind field is an unbalanced cyclonic vortex which increases with height and has a maximum surface value about $2 \mathrm{~m} / \mathrm{sec}$. Except for the tangential wind field, the initial value in the experiment B is same as that in the experiment A.

\section{The results of experiments}

The tangential component changes very slowly and the radial component and vertical motion are very small during the early stage after time integretion of Expertment $\mathrm{A}$. The tangential component at $24 \mathrm{hr}$ is shown in Fig. 4.1. A weak vortex with a maximum value of $6 \mathrm{~m} / \mathrm{sec}$ on $300 \mathrm{mb}$ level and at 200 $\mathrm{km}$ from the center is produced. At the same time, the initial temperature is changed very little. It shows that an imbalance field is forced by $\mathrm{Fe}$ at first. The tangential and radial speed begin to develop at $30 \mathrm{~h}$. It can be seen from Fig. 4.2 (a) that outflow in the upper levels and inflow in the low levels develop. The horizontal scale of the vertical motion shown in Fig. 4.2 (b) is about $50-100$ $\mathrm{km}$, which is approximately equivalent to the horizontal scale of tropical cloud clusters. Heat and moist static energy are transferred from lower to upper levels by these deep convective systems. The vertical motion at the center is upward during the early stage of the development. This can be seen from the precipitation for $0-36 \mathrm{~h}$ shown in Fig. 4.3.

In order to test whether the $F e$ is important or not after the experiment has been conducted for some time, say $36 \mathrm{~h}$, two different experiments are examined. The experiment which $F e$ remains after $36 \mathrm{~h}$ is denoted by $\mathrm{A} 1$ and the expriment in which $\mathrm{Fe}$ is set to be zero after $36 \mathrm{hr}$ is called A2. The tangential and radial speed and the

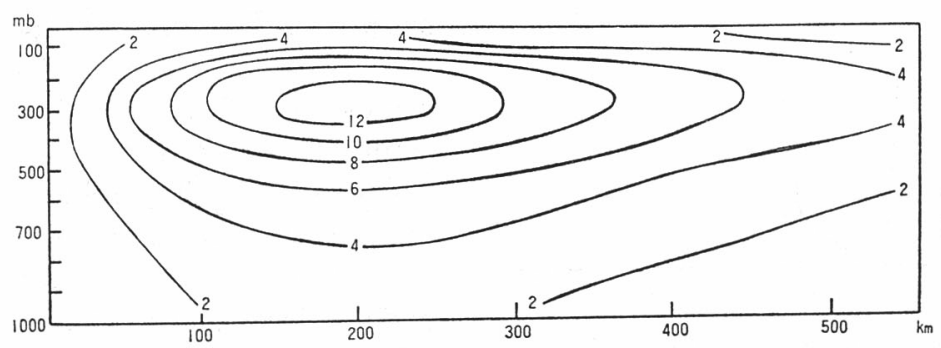

Fig. 3.2 The distribution of the initial imbalanced tangential wind used in the experiment B. (Unit: $\mathrm{m} / \mathrm{s}$ ) 


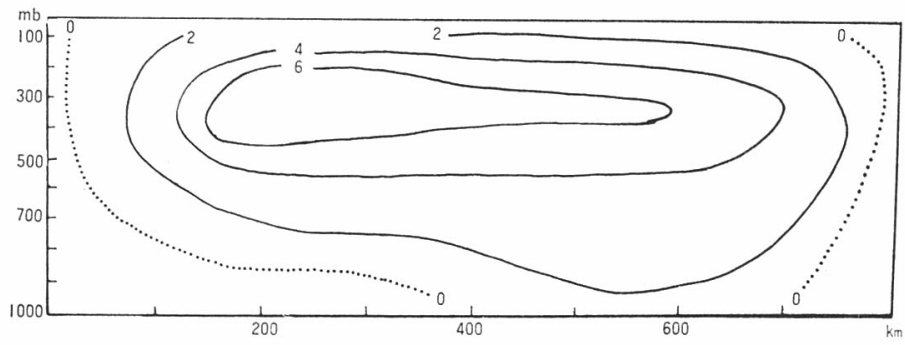

Fig. 4.1 The distribution of the tangential speed at $24 \mathrm{~h}$ after the initial moment
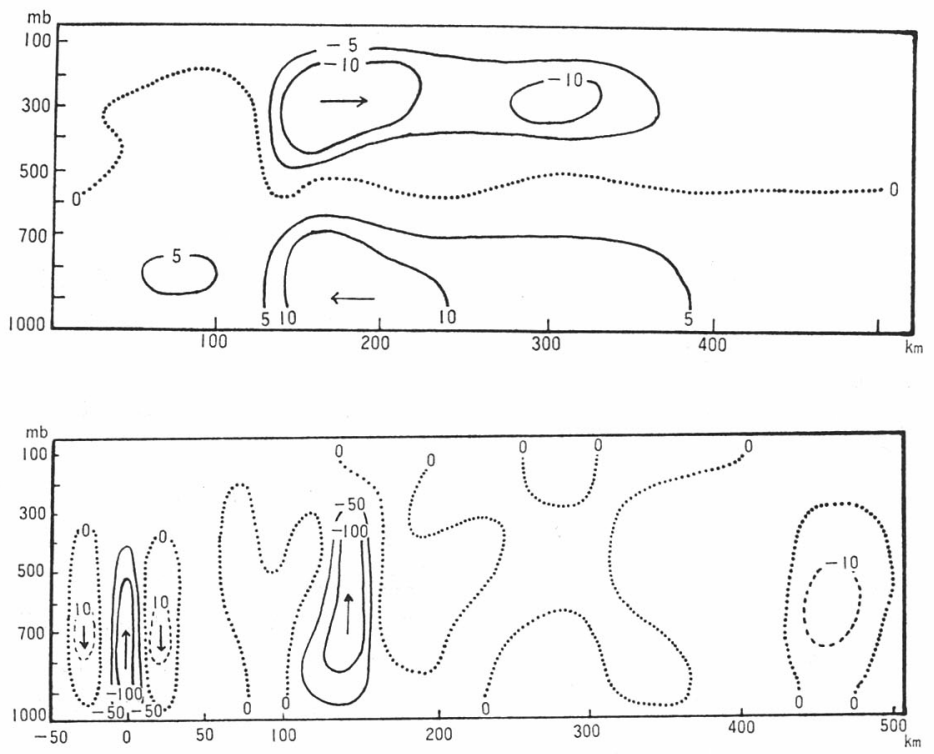

Fig. 4.2 The distribution at $30 \mathrm{~h}$ (a) radial wind speed (Unit: $\mathrm{m} / \mathrm{s}$ ) and (b) the vertical velocity (Unit: $10^{-3} \mathrm{mb} / \mathrm{s}$ ).

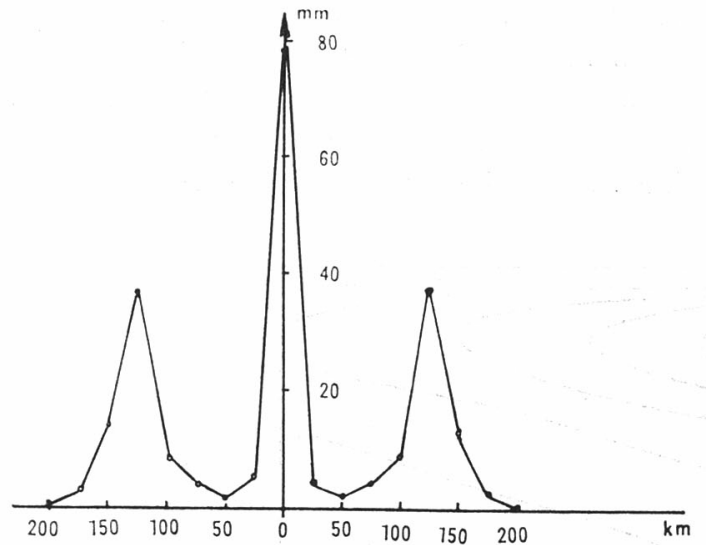

Fig. 4.3 The distribution of the total precipition of $0-36 \mathrm{~h}$. vertical motion at $48 \mathrm{~h}$ for experiment $\mathrm{A} 1$ are shown in Fig. 4.4(a)-(c) respectively. The maximum surface tangential speed in Fig. 4.4 (a) is $40 \mathrm{~m} / \mathrm{see}$ and equivalent to the intensity of a typhoon. In addition to the primary inflow maxima in the boundary layer shown in Fig. 4.4(b), there is a secondary maxima of inflow near $400-500 \mathrm{mb}$ beyond a radius of $150 \mathrm{~km}$. This phenomennon is similar to the observed result shown in Fig. 1.3 , and is possibly related to the influence of the environmental flow. The vertical motion in Fig. 4.4 (c) is further systematized, and the decending motion occurs in the center. The maximum ascending motion takes place near the radius of $50 \mathrm{~km}$, so that an eye and 

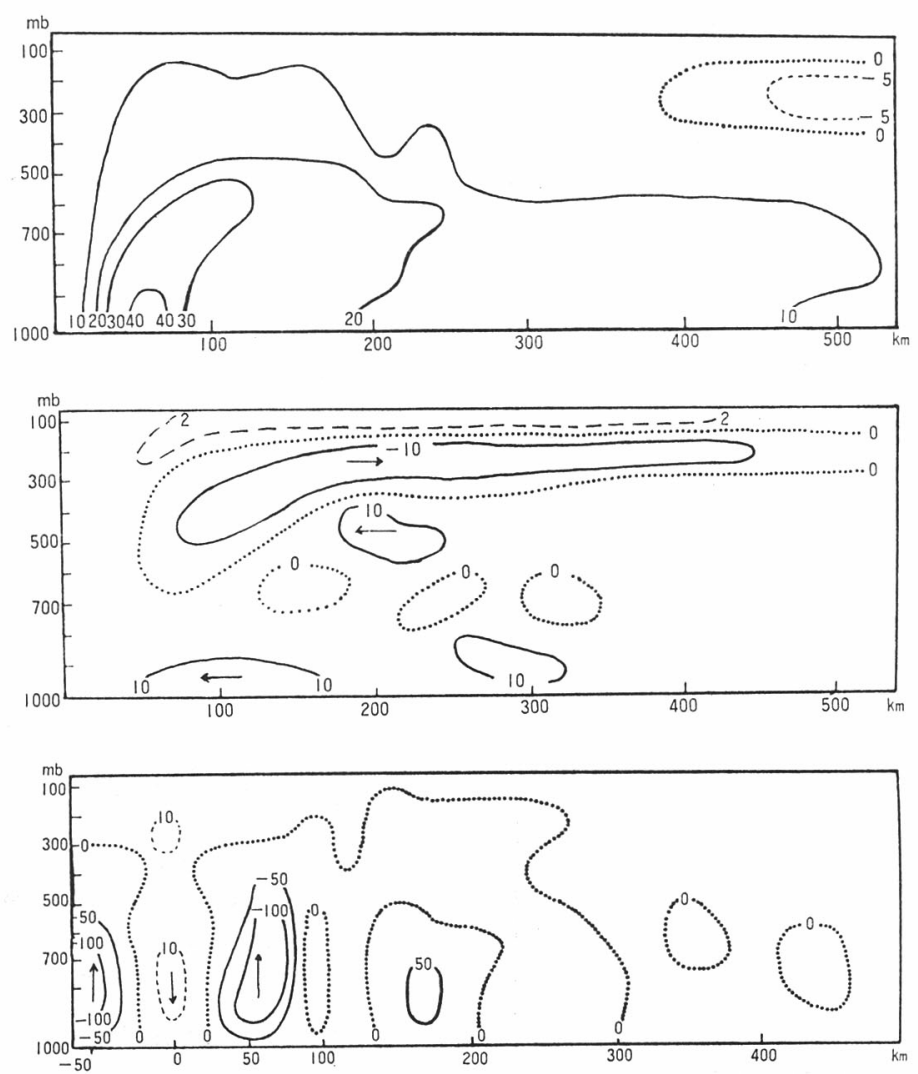

Fig. 4.4. The distribution of the experiment $\mathrm{Al}$ at $48 \mathrm{~h}$ : (a) tangental wind speed (Unit: m/s), (b) and (c) same as Fig. 4.2 (a) and (b).

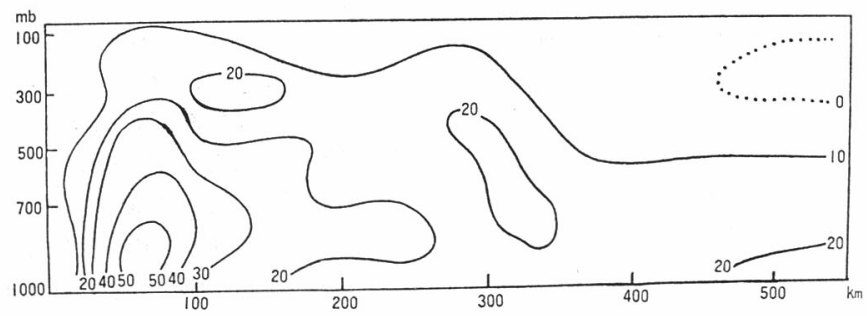

Fig. 4.5 The distribution of the tangential wind speed at $54 \mathrm{~h}$ for the experiment A1.

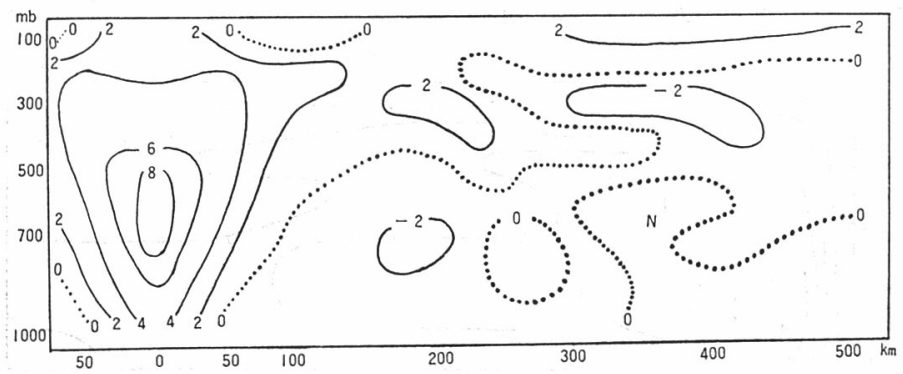

Fig. 4.6 The distribution of the deviation of the radial mean temperature at $54 \mathrm{~h}$ for the experiment $\mathrm{A} 1$ (Unit: ${ }^{\circ} \mathrm{C}$ ). 
eye-wall develop in the model typhoon. In the region out of eye-wall, decending and ascending motion develop alternatively. Their scale is about $100 \mathrm{~km}$ and resembles the spiral bands in three-dimensional models or in real typhoons. The model typhoon continues to develop after $48 \mathrm{~h}$, and maximum tangential speed is $55 \mathrm{~m} / \mathrm{sec}$ at $54 \mathrm{~h}$ shown in Fig. 4.5 . The deviation of the radial mean temperature at $54 \mathrm{~h}$ is shown in Fig. 4.6, it can be seen that the whole tropsphere is a warm center in the eye region. The profile of the surface presure at $54 \mathrm{~h}$ is shown in Fig. 4.7 and the minimum pressure in the center is $960 \mathrm{mb}$. The precipitation for $36-60 \mathrm{~h}$ shown

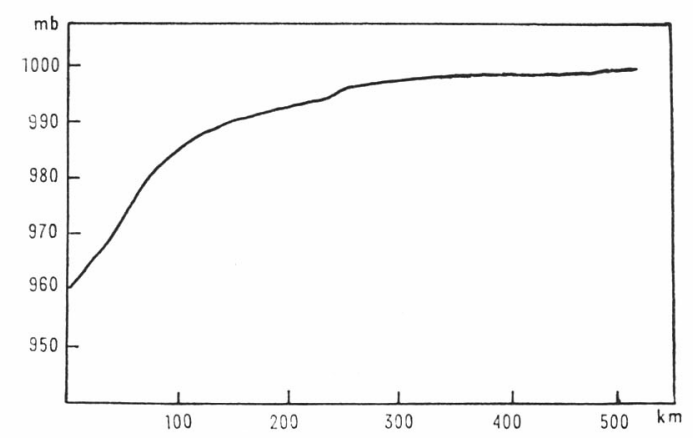

Fig. 4.7 The profile of the surface pressure at $54 \mathrm{~h}$ for the experiment A1.

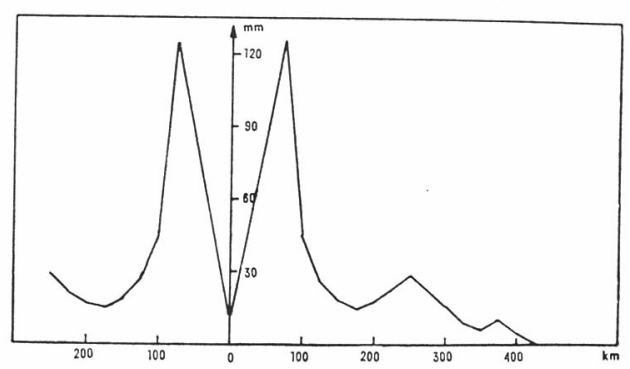

Fig. 8. The distribution of the total precipitation of $26-€ 0 \mathrm{~h}$ for the $\epsilon$ xferinet.t A1. (Lrit: $\mathrm{mm}$ ) in Fig. 4.8 is quite different from that for $0-36 \mathrm{~h}$ in Fig. 4.3. Most of the precipitation occurs near the eye-wall at the radius about $50-100 \mathrm{~km}$; very little occurs in the center.

The maximum surface tangential speed for experiment $\mathrm{A} 2$ at $54 \mathrm{~h}$ is $57 \mathrm{~m} / \mathrm{sec}$ shown in Fig. 4.9. The minimum surface pressure in the center at $54 \mathrm{~h}$ for $\mathrm{A} 2$ is $958 \mathrm{mb}$. The precipitation of A2 for $36-60 \mathrm{~h}$ is shown in Fig. 4.10. No precipitation occurs in the eye region; the maximum rainfall is concentrated near the eye-wall region. Comparing the experiments $\mathrm{A} 1$ and $\mathrm{A} 2$, the experiment $\mathrm{A} 2$ is quite similar to A1 after $36 \mathrm{~h}$, so that the effect of environmental flow on the formation of the typhoon is important only in the early stage.

As for results of experiment $B$ based on the initial implicit imbalance, the distribution of the vertical motion at $12 \mathrm{~h}$ is shown in Fig. 4.11, it can be seen that the maximum ascending motion occurs at the radius of 50 $\mathrm{km}$, and an eye begins to develop at the center as shown by the descending motion in the upper troposphere. The maximum tangential speed increases to $50 \mathrm{~m} / \mathrm{sec}$ at $24 \mathrm{~h}$ at a radius of $50 \mathrm{~km}$ shown in Fig. 4.12. The descending motion develops to the lower troposphere in the eye region at $24 \mathrm{~h}$ as shown in Fig. 4.13. The surface pressure at the center at $24 \mathrm{~h}$ is $962 \mathrm{mb}$. The deviation from the radial mean temperature at $24 \mathrm{~h}$ is shown in Fig. 4.14. A warm core develops in the eye region and the thermal structure is quite similar to the observed typhoon. The distribution of the radial speed at $36 \mathrm{~h}$ is shown in Fig. 4.15; there are two maxima of inflow in the region beyond the eye-wall. One is within the boundary layer, the other one is about $400 \mathrm{mb}$ level, which is also quite similar

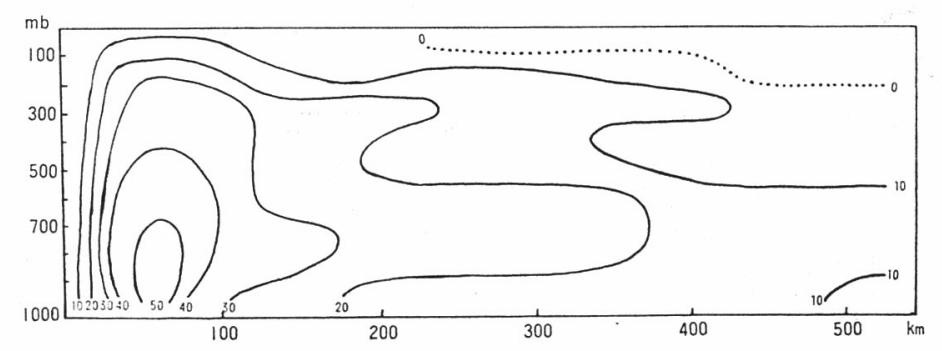

Fig. 4.9 Same as Fig. 4.5 but for the experiment A2. 


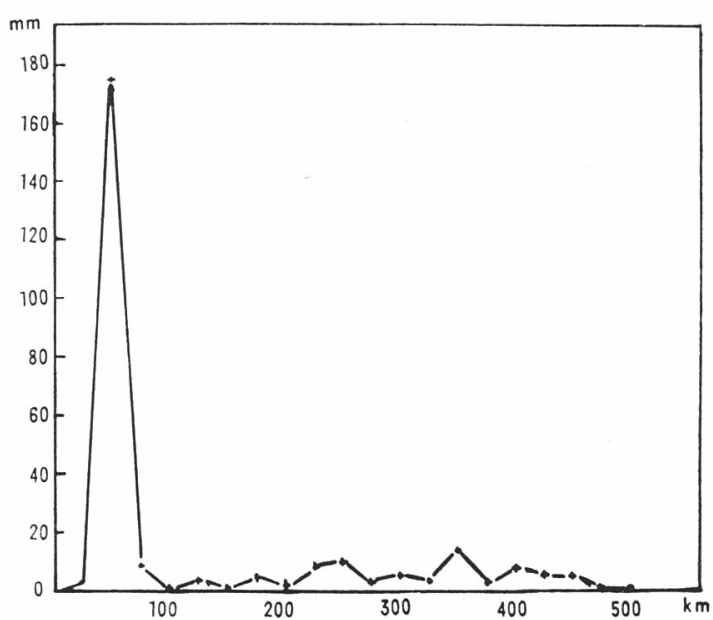

Fig. 4.10 Same as Fig. 4.8 but for the experiment A2. to composite typhoon shown in Fig. 1.3. The accumulated precipitation is shown in Fig. 4.16. The maximum rainfall develops near the eye-wall at the radius of $50 \mathrm{~km}$. The total precipitation for $24 \mathrm{~h}$ and $36 \mathrm{~h}$ are $112 \mathrm{~mm}$ and $261 \mathrm{~mm}$ respectively at the eyewall region.

Compared to the results of experiment $A$, because the influence of the environmental flow is concentrated on the initial moment, the model typhoon develops in experiment $B$ more quickly than in experiment $A$, and the eye and eye-wall in experiment $B$ are better developed than in experiment A. Therefore, the results show that the specific implicit imbalance, such as shown in Fig. 3.2, triggers the genesis and development of typhoon very

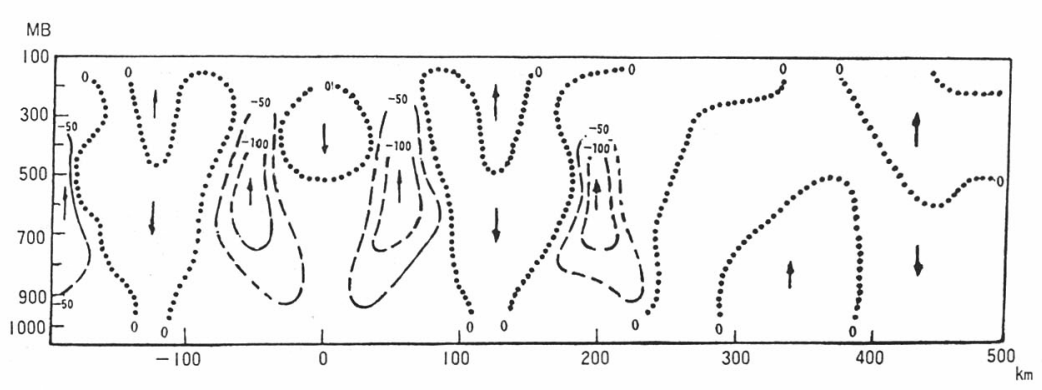

Fig. 4.11 The distribution of the vertical motion at $12 \mathrm{~h}$ (Unit: $10^{-3} \mathrm{mb} / \mathrm{s}$ ).

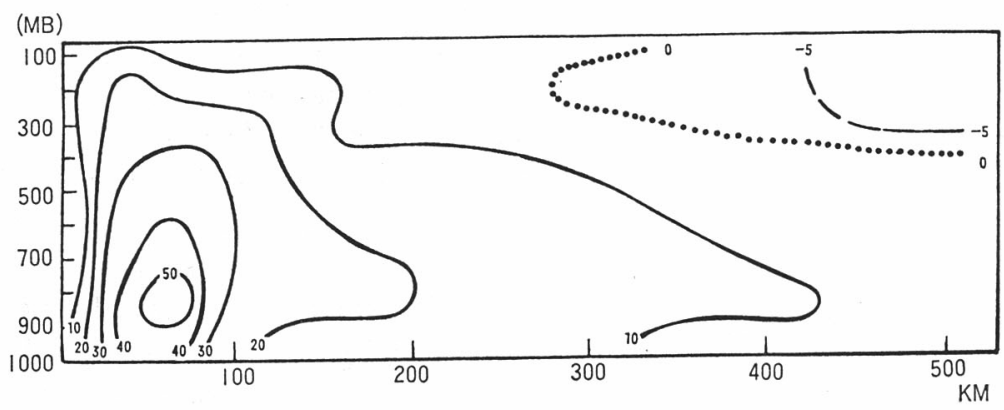

Fig. 4.12 The distribution of the tangential wind speed at $24 \mathrm{~h}$ (Unit: $\mathrm{m} / \mathrm{s}$ )

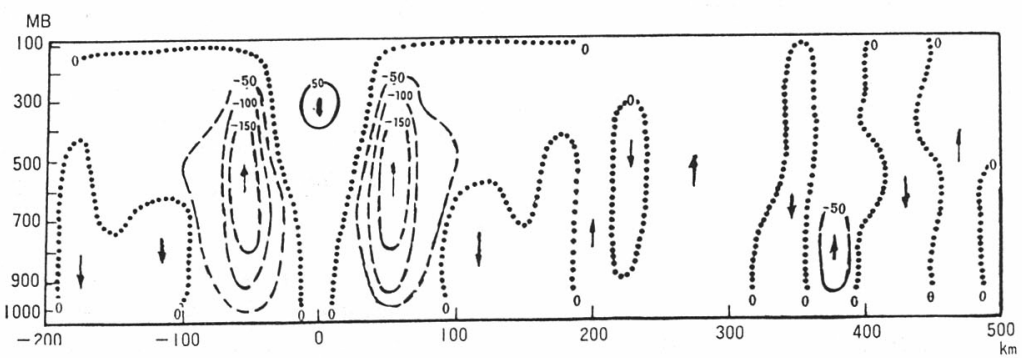

Fig. 4.13 Same as Fig. 4.11 but at $24 \mathrm{~h}$. 


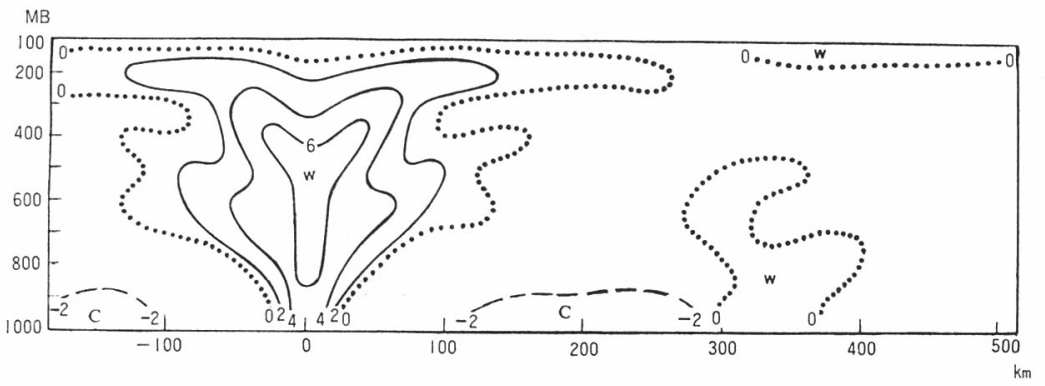

Fig. 4.14 The distribution of the deviation of the radial mean temperature at $24 \mathrm{~h}$.

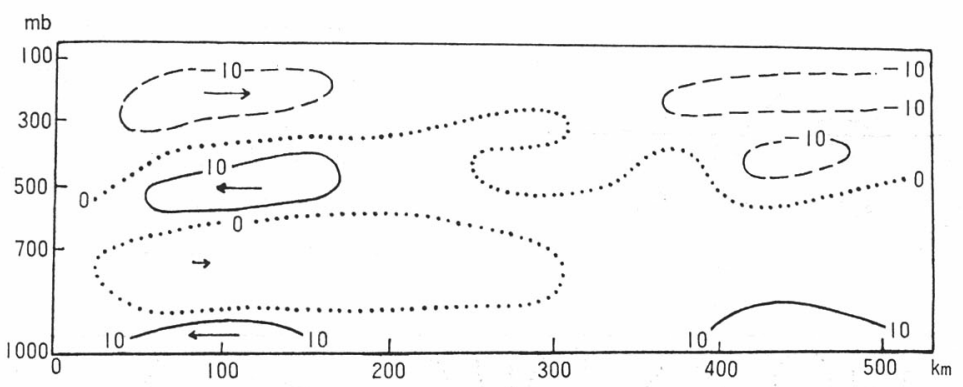

Fig. 4.15 The distribution of the radial wind speed at $36 \mathrm{~h}$ (Unit: $\mathrm{m} / \mathrm{s}$ (

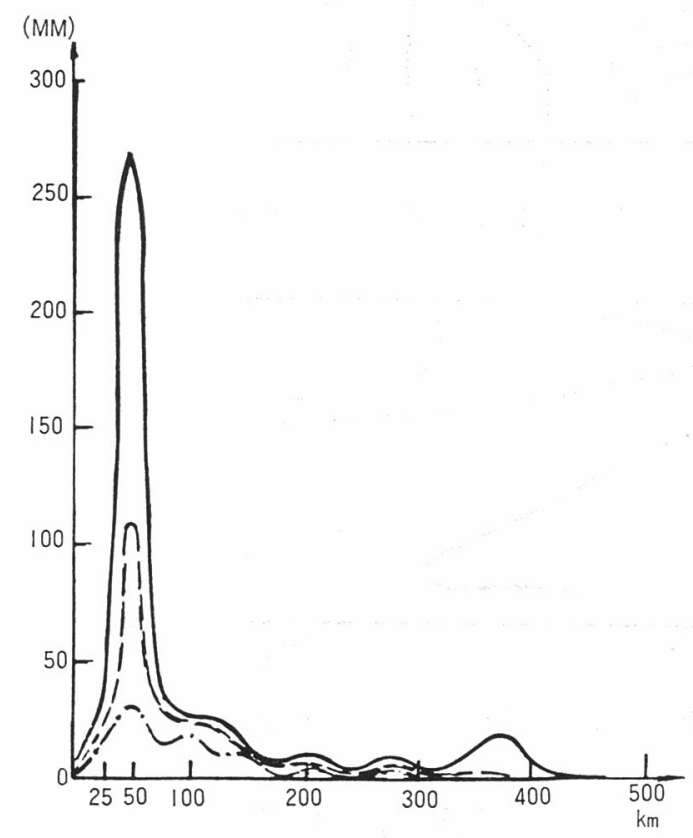

Fig. 4.16 The distribution of the total precipitation. (Solid line for $36 \mathrm{~h}$, dashed line for $24 \mathrm{~h}$, and dash-dotted line for $12 \mathrm{~h}$ respectively)

efficiently,

\section{Conclusion and remarks}

The above numerical experiments show that the genesis and development of the typhoon are strongly influenced by the environmental flow.

1. If a source term of cyclonic momentum, related to the eddy flux of momentum in an asymmetric environment, is added to the tangential equation of motion in the upper troposphere, and certain conditions for the supply of water vapor, sea surface temperature, the conditionally unstably static stratification and so on are satisfied, a typhoon will develop. The model characteristics of the eye, eye-wall and the distribution of the tangential and radial wind speed and precipitation, especially the vertical distribution of mass inflow are all quite similar to those in observed typhoons. The secondary maxima of mass inflow near $400 \mathrm{mb}$ level can be explained by modeling of the influence of the environmental flow, which was recently discussed by Holland and Merril (1984).

2. The influence of the environmental flow discussed above is important only in the early stages. For an example, if an influence in a certain strength exists only for first $36 \mathrm{~h}$, the genesis and development of the typhoon can also occur in the modeling.

3. From the physical analysis by the 
splitting method, the dynamical essentials of the influence of the environmental flow is its advection to cause an implicit imbalanced vortex, of which the vorticity of the shear wind is larger than the thermal vorticity computed from the temperature field, in the advection process. This implicit imbalance can be an initial condition to force a typhoon scale disturbance to grow into a typhoon very rapidly in the adjustment process. The simulated results are further illustrated and tested the theoretical results based on linear study (Chen, 1964). If the specific implicit imbalanced vortex is used as initial values instead of the forcing term due to the invironmental flow in the governing equation, the typhoon will generate and develop more quickly and clearly in the model.

The present paper is only a first step to study the influence of the environmental flow on the development of the typhoon. An asymmetric model should be used in further studies, and observed environmental flow should be used as initial values so that the imbalanced fields are automatically caused by environmental flow itself.

It should be mentioned that if we view the modeling based on CISK theory with an initial balanced vortex from the splitting method, the surface friction can be included in the advection process. The friction in the boundary layer will reduce the cyclonic surface wind so that a specific imbalance, in which the vorticity of the vertical shear wind is larger than the thermal vorticity computed from the temperature field, can also be caused in the advection process. Therefore, the triggering mechanism also implicitly exists in the numerical modeling based on CISK theory. However, in the real atmosphere, the CISK is not a unique mechanism to trigger the formation of the typhoon. It seems likely that many other processes, such as the influence of the environmental flow, can result in the genesis and development of the typhoon efficiently, and CISK and the forcing by the environmental flow could also both be working at the same time. It should make remarks on that the values of $\mathrm{Fe}$ are taken from the data for intensifying tropical cyclones and the observed flux may be resulted not only from the environmental flow but also from the asymmetry which develop in an intensitying vortex. It should further be studied in a three-dimensional model.

\section{Acknowledgement}

The author wishes to acknowledge Dr. R. A. Anthes for his review of the manuscript to result in numerous improvements, including his revision in English, to the final paper. The author thanks very much to Ms. Moyin Wang and Mr. Guanchen Dong for their helping to do some calculations on computers during the preparation of the manuscript.

\section{References}

Black, P. G. and R. A. Anthes, 1971: On the asymmetric structure of the cyclone outflow layer. J. Atmos. Sci., 28, 1348-1366.

Brown, J. and K. Campara, 1978: An economical time-differencing system for numerical weather prediction. Mon. Wea. Rev., 106, 1125-1136.

Challa, M. and R.L. Pfeffer, 1980: Effects of eddy fluxes of angular momentum on model hurricane development. J. Atmos. Sci., 37, 1603-1618.

Chen, Q., 1964: The instability of the gravityinertia wave and physical analysis of the formation of the typhoon in the early stage. Acta. Met. Sinica., 34 433-442 (in Chinese).

and J. Zhong, 1965: The synoptical analysis of the effects of the large-scale circulation on the formation of typhoons. Acta. Met. Sinica., 36, 486-497. (in Chinese)

, 1981: The physical mechanism of baroclinic instability and its downstream propagation based on the splitting method. Mon. Wea. Rev.., 109, 2464-2473.

- 1987: An axisymmetric primitive equation model and the numerical experiment of the formation of typhoon. Acta. Met. Sinica. (to be published).

Gray, W.M., 1977: Huricanes: Their formation, structure and likely role in the tropical circulation. Meteorology over the tropical oceans. Roy. Met. Soc. Billiny and Sons Limited, 155218.

218. E. Ruprecht and R. Phelps, 1975: Relative humidity in tropical weather systems. Mon. Wea. Rev., 103, 685-690.

Holland, G.J., 1983a: Angular momentum transports in tropical cyclones. Quart. J. Roy. Met. Soc., 109, 187-209. , 1983b: Tropical cyclones in the Aust- 
ralian/South west Pacific region. Dept. of Atmos. Sci. Paper, No. 363, Colo, State Univ. and R. Merril, 1984: On the dynamics of tropical cyclone structure changes. Quart. J. Roy. Met. Soc., 110, 723-745.

Matsuno, T., 1966: Numerical integretions of the primitive equations by a simulated backward difference method. J. Met. Soc. Japan, 44, 7684.

Orlanski, I., 1976: A simple boundary condition for the unbounded hyperbolic flow. J. Comput. Phys., 21, 251-269.
Rosenthal, S.L., 1978: Numerical simulation of tropical cyclone development with latent heat release by resolvable scales I: model description and preliminary results. J. Atmos. Sci., 35, 258-271.

Sundqvist, H., 1970: Numerical simulation of the development of tropical cyclones with a tenlevel model. Part 1. Tellus, 22, 369-390.

Yamasaki, M., 1977: A preliminary experiment of tropical cyclone without parameterizing effects of cumulus convection. J. Met. Soc. Japan, 55, 11-30. 